\title{
IS THE ENUCLEATION PROCESS AS A TREATMENT MODALITY FOR AGGRESSIVE (LARGE-SIZED) CEMENTO-OSSIFYING FIBROMA OF THE MAXILLA CURING ?
}

\author{
Wael A. Elmohandes*, Salah M. Elnady** and Salem Waly**
}

\begin{abstract}
The purpose: Is to assess the efficacy of enucleation process as an effective treatment modality in management of large sized (aggressive) cemento-ossifying fibroma (COF) or not.

Patients and Methods: Six patients presented with a large expansile, painless growth in the maxilla, diagnosed a cemento-ossifying fibroma were included in this study. The surgical techniques of COFs which applied for six cases were the enucleation of the tumor masses with surgical removal of the sclerotic margins around the lesions.
\end{abstract}

Results: Enucleation process was curative for (aggressive) large sized COF lesions. The interesting points; the capsules of lesions were not violated and the sclerotic margins were tumor free.

Conclusion: Enucleation with peripheral curettage is quite enough surgical intervention for management of large sized COFs. .

\section{INTRODUCTION}

Cementoossifying fibroma (COF) is considered a benign osseous tumor although, COF is closely related to other lesions such as fibrous dysplasia, cemetifying periapical dysplasia or cementoosseous florid dysplasia, COF is forming its own entity according to the 1992 classification of WHO. ${ }^{1}$ It is a bony tumor of the maxilla of possible odontogenic origin. It is believed to derive from the cells of the periodontal ligament. ${ }^{2,3}$
Radiologically, a well-defined radiolucency with or without sclerotic border is typically seen in COF. This radiographic appearance is often associated with jaw expansion, and these lesions may occasionally have an ill-defined border if relatively rapid growth occurs. Histopathologically, this type of COF shows scant bony trabeculae, with a predominance of cellularity in the stroma. The mixture of radiolucency and opacity is the second most common appearance of $\mathrm{COF}$ and almost always has a well-defined border with a variable

* Assistant Professor of Oral and Maxillofacial Surgery, Faculty Of Dentistry, Al-Azhar University.

** Lecturer of Oral And Maxillofacial Surgery, Faculty of Dentistry Al-Azhar University. 
degree of jaw expansion. A fibrous capsule or an attenuated layer of cortical bone may be present microscopically. A pure radiodensity is rarely encountered in $\mathrm{COF}$ and, when present, exhibits a homogeneous opacity with a very smooth outline. ${ }^{4}$

Occasionally, COF may grow to massive size and cause serious cosmetic and functional problems. Rarely, reports of COF lesions behaving in an aggressive fashion, diagnosed as 'aggressive', 'juvenile', or 'active' COF, present in the literature. At the present time, the relationship of these 'aggressive' lesions to the 'usual' COFs is unclear; whether they actually represent different lesions or are simply variants of COFs needs careful documentation of additional cases. ${ }^{5,6}$

The surgical management techniques of COFs are conservative ${ }^{7}$ and include enucleation with curettage in the case of small lesions, but may require radical modalities of management when they are aggressive and produce functional disturbances. ${ }^{8-11}$ The purpose of this study is to assess the efficacy of enucleation with peripheral curettage as an effective treatment modality in management of large sized (aggressive) COF or not.

\section{PATIENTS AND METHODS}

Six patients presented with a large expansile, painless growth in the maxilla, diagnosed a cemento-ossifying fibroma were included in this study. The diagnosis protocol contained clinical examination, radiographic evaluation by using panoramic x-ray and computed tomography (CT) imaging. Aspiration and incisional biopsies were made to confirm the diagnosis.

Clinical examination was made to assess size of lesion, disfigurement of facial structures, presence of intra-oral mucosal ulcer, and presence of bleeding (fig. $1 \mathrm{a}$ ).

Radiographically; panoramic x-ray was done to overview site, size, and shape of lesion. CT scan was made preoperatively to look the extension of lesion to the surrounding structures, as well as, nature of lesion (fig.1-b). CT scan was done 6 months postsurgically to assess remaining bony structure, and surgical field for recurrence.

Aspiration biopsy and incisional biopsy were made preoperatively to help in lesion diagnosis.

The surgical techniques of COFs which applied for six cases was the enucleation of the tumor mass with surgical removal of the sclerotic margins around the lesion separately and each specimen (lesion and sclerotic margins) studied histopathologically.

The patients were prepared for the surgery. The general anesthesia was used for all cases. A total maxilla exposure was performed with a WeberFerguson cheek flap approach. The lesion was exposed in most directions and the osteotomy cuts were marked on the bone to expose the lesion in other directions. In some cases, a palatal flap was raised and the palatal cuts were done. In large sized aggressive cases, the lateral orbital rim was exposed and the temporal process of the zygoma was also visualized. When the orbit was involved, the orbital floor was dissected subperiosteally and the globe retracted superiorly visualizing the entire orbital floor. The lesion was enucleated at the most accessible areas, the nasal bone, then, going down to the alveolar process. The enucleation continues laterally to the zygoma, superiorly to the orbit cautiously to avoid any hurt to the globe. By using the clamp (holding instrument), the lesion would be dislodged minimally to define the area of resistance, finally the lesion was removed in one piece after removal the obstacles(fig. $1 \mathrm{c}$ ). The sclerotic margins (the bony surrounding) were excised separately to be examined as another biopsy tissue. The oral layer was sutured primarily with an advancement of the cheek flap to the dissected palatal flap. The cheek flap was sutured in layers with 4-0 vicryl and 4-0 nylon sutures in an interrupted fashion (fig. $1 \mathrm{~d}$ ). 


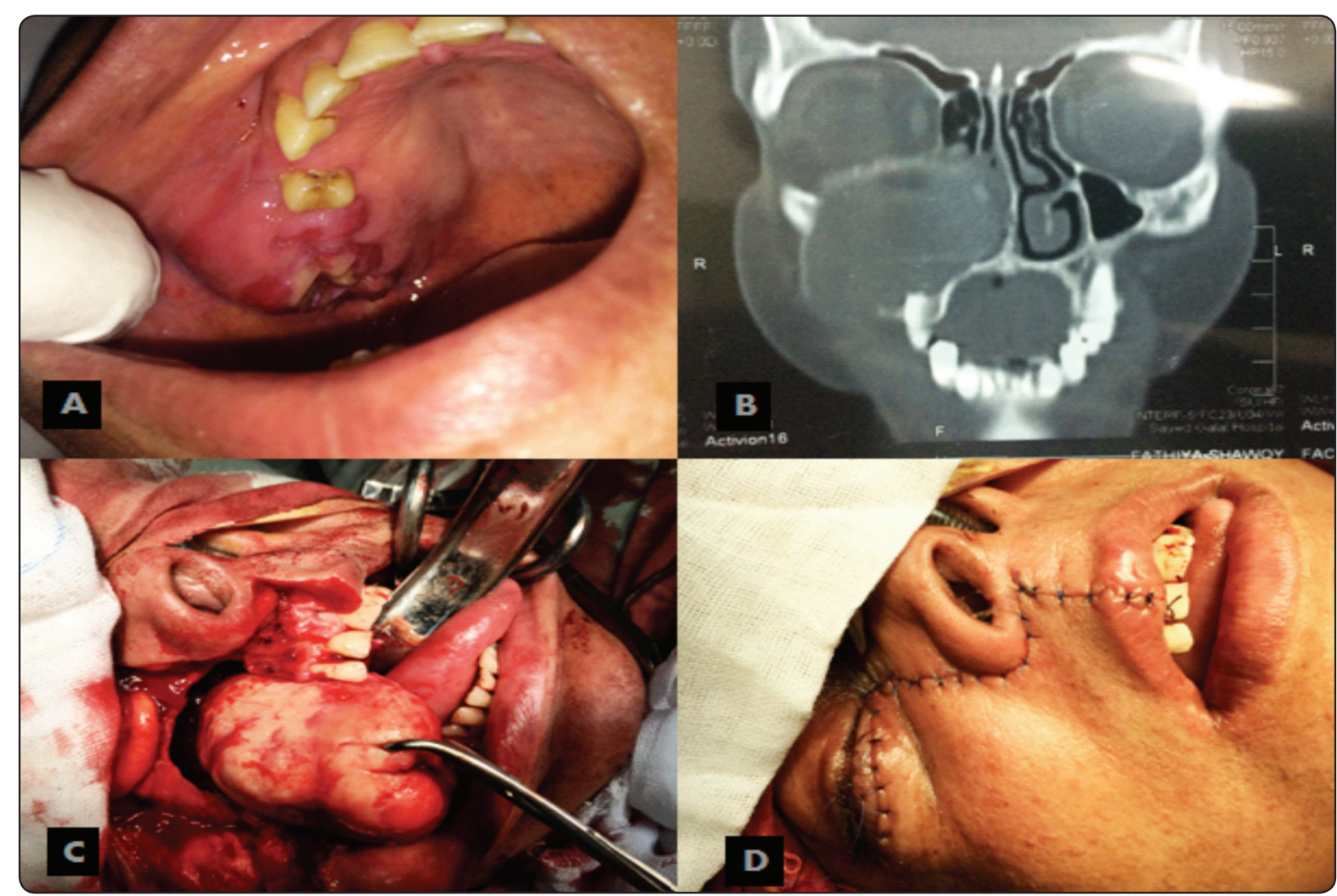

Fig. (1) a: Shows swelling preoperatively. b:shows radiographic appearance of the lesion. C: shows intraoperative lesion size. D: shows skin suturing after lesion removal.

\section{RESULTS}

The follow up period was continued for one year postoperatively with making CT 6 months postoperatively. In this article, six patients aged from 33 to 63 years with mean age 49.6 years. The large aggressive lesions were diagnosed as the cemento-ossifying fibroma by incisional biopsy. The enucleation with peripheral curettage was used as a treatment modality in all cases.

The recovery after the surgery was uneventful. Postoperative review of the patients revealed $80 \%$ of patients showed moderate edema, $20 \%$ of patients showed mild edema.Postoperatively $60 \%$ of patients complained of moderate pain while the remaining patients showed mild pain and slight blood oozing which were controlled with medication and packing respectively. After the resolution of edema, minimal disfiguring as flattening of the side of the cheek owing to removal of the lesions was noticed. The reconstruction of the defect was differed according to each case, obturators, zygoma implants or bone grafting.

Clinical examination of many cases revealed a solitary swelling visible extraorally, measuring about (6 to $9 \mathrm{~cm}$ mean 7.7). Intraoral examination revealed soft tissue swelling, firm in consisitency and obliterating the adjacent vestibule. The swelling in some cases wasn't tender. The overlying skin or the mucosa did not present any secondary changes like ulceration or spontaneous bleeding. Three cases showed significant proptosis of the corresponding eye without any ophthalmic impairment. There was no anaesthesia or paraesthesia of the adjacent nerves. 
Panoramic imaging studies demonstrated a radiopaque mass in the maxillae with expansive remodeling of bone and focal loss of cortical bone. The mass invaded maxillary sinus. It was wellcircumscribed and showed radiolucent and radioopaque features. In most cases, CT scan revealed a large intra-osseous mixed density mass with diffuse scattered calcification involving the maxillary alveolar ridge, occupying and expanding the maxillary sinus, reaching the pterygoid plates and displaced the orbital floor superiorly.

Aspiration showed negative results, ruling out cystic lesion, and then an incisional biopsy was performed under local anesthesia from the lesion and sent for histopathological examination. The histopathological study showed fibrous connective tissue with bone trabeculae and small, rounded, calcified foci that grouped into lobulated masses. COF was diagnosed, and patient underwent surgical intervention.

In all cases, the surgical specimens (lesions and sclerotic borders) were submitted for histopathological study. The results of reports showing the lesions were COF, which correlated with the earlier biopsy report. The interesting points, the capsules of lesions were not violated and the sclerotic margins were tumor free.

\section{DISCUSSION}

Benign fibro-osseous lesions are a poorly defined lesions and to some extent controversial group of lesions affect the jaws and craniofacial bones. Fibro-osseous lesions refer to a group of pathologic processes in which normal bone is replaced by fibroblasts and collagen fibers containing variable amounts of mineralized material. This group encompasses fibrous dysplasia, benign fibroosseous neoplasms (central ossifying fibroma), and a heterogeneous group of reactive lesions (osseous dysplasias). Because of the histopathologic similarities among these lesions, the definitive diagnosis requires a precise correlation of the clinical, histopathologic, and imaging findings. ${ }^{12,13}$
Although more lesions often found in the mandible, huge lesions of the maxilla are reported. The presence of orbital involvement is documented due to large lesions, more often resulting from delayed diagnosis.Sudden increase in size, secondary to surgery or trauma have also been described. ${ }^{14}$ The description of aggressive juvenile variety and psammomatoid varieties have been documented, which necessitate radical surgical management with preservation of function whenever possible. ${ }^{14}$ Large lesions involving the midface extending to the orbit may lead to functional disturbances like diplopia or progressive loss of vision as a result of optic nerve atrophy. These lesions need multispeciality management and extensive exposure for removal of the lesion. ${ }^{8,9}$

The clinical management of OF remains uncertain. Small lesions can be treated conservatively by curettage or enucleation, until bony healthy margins are reached..$^{15,16}$ On the other hand, larger lesions will require more radical surgical resection. ${ }^{7}$ Complete removal of the lesion at the earliest possible stage has been suggested by majority of the authors. ${ }^{17,18}$ Because Central OFs are well circumscribed, they usually "shell out" easily at surgery, but maxillary central OFs are more difficult to remove completely than mandibular central OFs. This may be attributable to the difference in bone character between the mandible and maxilla and to the available space for expansion in the maxillary sinus. ${ }^{19}$

Nature of midface bony structures as well as presence of maxillary air sinus in this region allow the tumor to expand largely in short time without patients feeling which makes lesion discovery late. This makes the treatment plan more difficult and need careful maneuver to preserve the surrounding vital structure.

Histopathologic examination for rarefied bone (surrounding marginal bone) revealed that there is no involvement of this surrounding bone by tumor cells which means that enucleation of the lesion with 
peripheral curettage could be quite enough surgical intervention for complete curing from this lesion.

Postoperatively; the patients revealed mild to moderate pain due to non-aggressive surgical approach. This non-aggressive approach was fastly (in short time) curing technique for the lesion.

One year follow up period for all patients of this study showed complete curing with no recurrence through this year which confirmed the efficacy of our surgical technique for complete curing.

\section{CONCLUSION}

Enucleation with peripheral curettage is quite enough surgical intervention for management of large sized COFs with preserving manipulation to the surrounding structure .

\section{REFERENCES}

1. Dehner LP. Tumors of the mandible and maxilla in children. Clinicopathologic study of 46 histologically benign lesions. Cancer 1973;31:364-71.

2. Khanna S, Khanna NN. Primary tumors of the jaws in children.J Oral Surg 1979;37:800-4.

3. Eversole LR, Leider AS, Nelson K. Ossifying fibroma: a clinicopathologic study of sixty-four cases. Oral Surg Oral Med Oral Pathol 1985;60:505-11.

4. Su L, Weathers DR, Waldron CA. Distinguishing features of focal cementoosseous dysplasias and cemento-ossifying fibromas. A clinical and radiological spectrum of 316 cases. Oral Surg Oral Med Oral Pathol Oral Radiol Endod 1997;84:540-9.

5. Summerlin DJ, Tomich C. Focal cemento-osseous displasia: a clinicopathologic study of 221 cases. Oral Surg Oral Med Oral Pathol 1984;78:611-20.

6. Zupi A, Ruggiero A, Insabato L, Senghore N, Califano L. Aggressive cemento-ossifying fibroma of the jaws. Oral Oncology $2000 ; 36 ; 129-133$.
7. Brannon RB, Fowler CB. Benign fibro-osseous lesions: a review of current concepts. Adv Anat Pathol 2001; 8:126-43.

8. John LF, Gerry F, Jay B, Mark R. Management of aggressive midface and orbital fibrous dysplasia. Arch Facial Plast Surg 2000;2:187-95.

9. Nakagawa K, Takasato Y, Ito Y, Yamada K. Ossifying fibroma involving the paranasal sinuses, orbit and anterior cranial fossa: case report. Neurosurgery 1995;36:1192-5.

10. Pasquale C, Roberto D, Giorgio I, Maurizio S, Antonio R. Surgical strategies in the treatment of symptomatic osteomas of the orbital walls. Neurosurgery 1992;31:628-35.

11. Salyer KE, Barcelo CR, Por YC. Extensive neglected psammomatoid ossifying fibroma with craniofacial deformity. J Craniofac Surg 2004;15:1033-9.

12. Speight PM, Carlos R. Maxillofacial fibro-osseous lesions. Curr Diagn Pathol 2006;12:1-10.

13. Eversole R, Su L, ElMofty S. Benign fibro-osseous lesions of the craniofacial complex. A review. Head Neck Pathol 2008;2:177-202.

14- Bowyer JD, Majid MA, Ah-Fat F, Kaye SB, Kokai GK, May PL, et al. Giant cemento-ossifying fibroma of the maxilla causing proptosis in a young patient. J Pediatr Ophthalmol Strabismus 2001;38:359-62.

15- Jung SL, Choi KH, Park YH, Song HC, Kwon MS. Cement-ossifying fibroma presenting as a mass of the parapharyngeal and masticator space. Am J Neuroradiol 1999;20:1744-6.

16- Kristensen S, Tveteras K. Aggressive cementifying fibroma of the maxilla. Arch Otolaryngol 1986;243:102-5.

17. Scholl RJ, Kellet HM, Neuman DP, Lurie AG. Cysts and cystic lesions of the mandible: clinical and radiologic-histopathological review. Radiographics 1999; 19:1107-24.

18- Kouri ME, Regezi JA, Perrott DH, Kaban LB. Atypical fibro-osseous lesions: Diagnostic challenges and treatment concepts. Int J Oral Maxillofac Surg 1995;24:162-9.

19- Artico M, Cervoni L, Salvata M, Raco A, Ciapetta P. Ossifying fibroma of the skull: Clinical and therapeutic study. Tumori 1994;80:64-7. 